\title{
ON THE DESIGN OF CHROMOSPHERIC MODELS
}

\author{
U. GROSSMANN-DOERTH
}

Fraunhofer Institut Feiburg, Germany

\begin{abstract}
The basic features of the so-called 'cloud-model' (Grossmann-Doerth and von Uexküll, 1971) are discussed. The model permits us to derive approximate values of certain properties of the elements of the chromospheric fine structure by comparing the observed profiles of strong Fraunhofer lines with a simple theoretical formula involving four free parameters. The method is only applicable if two basic conditions are met: (1) The absorption coefficient varies with wavelength according to a gaussian with a broadening parameter that is independent of height, (2) the variation of the source function $S_{v}$ with height is sufficiently small so that

$$
S(\lambda)=\int_{0}^{\tau} S_{v}(z) e^{-t} \mathrm{~d} t\left(\int_{0}^{\tau} e^{-t} \mathrm{~d} t\right)^{-1}
$$

may be replaced by a constant. The validity of these assumptions, in particular the latter one, is discussed. It is shown that in the case of $\mathrm{H} \alpha$ there are reasons to believe that the assumption is valid provided the features are not very close to the limb ( $\sin \theta=0.9$ may constitute the border line). There is also some evidence to suggest that the cloud model will not work for the Ca II K-line.

In the second part of the paper the presently used methods of constructing multicomponent models of the chromosphere are considered. It is proposed to apply the radiative transfer theory to selected parts of the chromosphere by comparing the model predictions with spatially resolved spectra and filtergrams instead of computing spatial averages and comparing those with integrated spectra.
\end{abstract}

\section{Reference}

Grossmann-Doerth, U. and von Uexküll, M.: 1971, Solar Phys. 20, 31.

\section{DISCUSSION}

Thomas: I am sorry to be negative on this paper. Jack Zirker and I looked at this thing about twelve years ago and we found then that Grossmann-Doerth's method cannot work.

Giovanelli: In the photograph that I showed earlier this morning, there were grains which have similar appearances to, and are rather larger than photospheric granules. These are in the centers of supergranule cells while there are other structures, the fibrils, that extend over them. The fibrils fade out towards the centers of the supergranule cells. I would expect the fibrils to be well described by a cloud model, but not the grains.

Grossmann-Doerth: I think I have not made myself very clear. I did not mean to suggest that we could apply the cloud model to the interior of supergranule cells; on the contrary, I said the cloud model is not applicable there. 